INPLASY

PROTOCOL

To cite: Hsu et al.

Heterogeneity and efficacy of mood stabilizer and atypical antipsychotic treatment for acute bipolar mania: a metaanalysis. Inplasy protocol 202210002. doi: 10.37766/inplasy2022.1.0002

Received: 01 January 2022

Published: 01 January 2022

Corresponding author:

Tien-Wei Hsu

twhsu@vghks.gov.tw

Author Affiliation:

Department of Psychiatry, Kaohsiung Veterans General Hospital, Kaohsiung, Taiwan.

Support: None.

Review Stage at time of this submission: The review has not yet started.

Conflicts of interest:

None declared.

\section{Heterogeneity and efficacy of mood stabilizer and atypical antipsychotic treatment for acute bipolar mania: a meta-analysis}

Hsu, TW1; Liang, CS2; Chu, CS³.

Review question / Objective: Heterogeneity and efficacy of mood stabilizer and atypical antipsychotics treatment to acute bipolar mania; P: acute mania; I: mood stabilizer or atypical antipsychotic monotherapy; C: placebo control; $\mathrm{O}$ : mania score; S:RCT.

Condition being studied: Several types of antipsychotics or mood stabilizers had been suggested as first-line treatment for bipolar mania. However, through comparison of the heterogeneity/mean of the efficacy of those medications to placebo, we could find the medication with high scores of mean difference and low heterogeneity compared with placebo, which indicates trustable treatment for bipolar mania.

INPLASY registration number: This protocol was registered with the International Platform of Registered Systematic Review and Meta-Analysis Protocols (INPLASY) on 01 January 2022 and was last updated on 01 January 2022 (registration number INPLASY202210002).

\section{INTRODUCTION}

Review question / Objective: Heterogeneity and efficacy of mood stabilizer and atypical antipsychotics treatment to acute bipolar mania; P: acute mania; I: mood stabilizer or atypical antipsychotic monotherapy; C: placebo control; O: mania score; S:RCT.

Condition being studied: Several types of antipsychotics or mood stabilizers had been suggested as first-line treatment for bipolar mania. However, through 
comparison of the heterogeneity/mean of the efficacy of those medications to placebo, we could find the medication with high scores of mean difference and low heterogeneity compared with placebo, which indicates trustable treatment for bipolar mania.

\section{METHODS}

Search strategy: We will search the EMBASE, The Cochrane Library, PubMed, MEDLINE, PsycINFO from the date of inception for randomized controlled trials without language restriction.

Participant or population: Patient with bipolar disorder, manic episode.

Intervention: Mood stabilizer or atypical antipsychotic monotherapy.

Comparator: Placebo control.

Study designs to be included: Placebocontrolled RCT.

Eligibility criteria: P: acute manial: mood stabilizer or atypical antipsychotic monotherapy C: placebo controlO: mania score S: RCT. from the date of inception for randomized controlled trials without language restriction.

Information sources: EMBASE, The Cochrane Library, PubMed, MEDLINE, PsycINFO.

Main outcome(s): mania scores, like YMRS or SADS-MRS (Variability ratio OR Coefficient of variability ratio).

\section{Additional outcome(s): (SMD OR Hedge's} g).

Quality assessment / Risk of bias analysis: Cochrane risk of bias 2.0.

Strategy of data synthesis: 1. standardized mean differences (SMD) between treatment groups were calculated for each trial and used to derive a total estimate of treatment effect on the outcomes. The SMD is a summary statistic in a meta-analysis when the studies assess the same outcome but with different scales. For interpretation of effect sizes, we followed the rules of classifying 0.8 as large. 2.Variability ratio OR Coefficient of variability ratio compared with placebo.

Subgroup analysis: Different types of drugs.

Sensitivity analysis: 1. subgroup analysis; 2. meta-regression.

Language: English.

Country(ies) involved: Taiwan.

Keywords: mood stabilizers; antipsychotics; heterogeneity of efficacy; bipolar mania.

Contributions of each author:

Author 1 - Tien-Wei Hsu.

Email: twhsu@vghks.gov.tw

Author 2 - Chih-Sung Liang.

Email: Icsyfw@gmail.com

Author 3 - Che-Sheng Chu.

Email: youngtzuchi@hotmail.com 